\title{
Site Occupation Preferences in CdMnTeSe Quaternary Alloys. EXAFS Data Analysis
}

\author{
A. Kisiel ${ }^{*}$, J. Lazewski***, M. Zimnal-Starnawska*, E. Burattini*** and A. Mycielski**** \\ * Institute of Physics, Jagellonian University, ul. Reymonta 4, 30-059 Kraków, Poland \\ ** Institute of Nuclear Physics, ul. Radzikowskiego 152, 31-342 Kraków, Poland \\ *** Laboratori Nazionali di Frascati, INFN, via E. Fermi 40, 00044 Frascati, Italy \\ **** Institute of Physics, Polish Academy of Science, Al. Lotników 32, 02-668 Warszawa, Poland
}

\begin{abstract}
Site occupation preferences in zinc-blende $C d_{1-x} M n_{x} T e_{1-y} S e_{y}(0.0 \leq x \leq 0.15 ; 0.0 \leq y \leq 0.20)$ quaternary alloys have been determined by the Extended X-ray Absorption Fine Structure (EXAFS) analysis. The experimental data have been collected with the use of synchrotron radiation for $S e$ - and $M n$ - $K$ edges. For both $K$ edges, the preferences of manganese distribution around selenium have been observed and analyzed. The $M n$-Se with $C d-S e$, and $M n-S e$ with $M n$-Te distances have been found as linearly dependent on concentration of $S e$ and $M n$. The average coordination numbers of $M n$ around $S e$ versus $M n$ composition was approximated by the quadratic behavior instead of the linear dependence expected for the virtual crystal approximation, in which a full random distribution of elements in the sublattices is assumed. The obtained results support the preferential occurring of $M n$ and $S e$ ions in the considered compounds. A structural and magnetic interaction reasons of the preferences were discussed.
\end{abstract}

Extended X-ray Absorption Fine Structure (EXAFS) analysis of the random ion distribution has been dated since the random cluster model (RCM) [1, 2] was applied to CdMnTe [1], \& $\imath C d T e, G a I n S b$ and $H g T e S e$ [3]. This model has been developed finally as a random iterative cluster model (RICM) by Wiedmann et al. [4] and applied to the analysis of the EXAFS data for $Z n_{1-x} M n_{x} S e$ [5]. In quaternary alloys site correlations are expected to be larger than for ternaries because nearest-neighbor preferences are possible. They were observed in InGaSbAs compounds by Islam and Bunker [6]. They found clear preference of $G a-A s$ pairs over $I n$-As pairs.

The aim of this paper is to analyze discrepancies in random distribution of quaternary $C d_{1-x} M n_{x} T e_{1-y} S e_{y}$ diluted magnetic semiconductors. Experimental $X$-ray absorption measurements have been carried out with use of synchrotron radiation at the Adone Wiggler facility in Frascati utilizing the Si(111) and $S i(220)$ channel-cut crystal monochromators [7]. The original samples were high purity monocrystalline binary $C d T e, C d S e, M n T e$ and $M n S e$ standard compounds, ternary $C d M n T e$ and $C d_{0.75} M n_{0.25} S e$ and $C d_{1-x} M n_{x} T e_{1-y} S e_{y}(z=0.00,0.05,0.10,0.15 ; y=$ $0.00,0.10,0.20)$ quaternary compounds produced by Bridgman method. The binary compounds crystallize in three different structures; i.e. $C d T e$ crystallize in zinc-blende structure, $M n T e$ in hexagonal NiAs structure, $C d S e$ in wurtzite structure and $\mathrm{MnSe}$ in $\mathrm{NaCl}$ structure. Quaternary compounds CdMnTeSe have well defined monophase zinc blende structure only for low $M n$ and $S e$ concentration. The phase diagram of this alloy system [8] shows wide two and three phase region in the center with narrow monostructure islands in the corners of phase diagram. Especially, the monophase zincblende region extends for $x<0.2$ when $y<0.2$ and $x<0.7$ when $y<0.05$. All compositions of studied samples derived from this monophase region. XAS measurements have been carried out on the $M n$ and $S e K$ edges. The contribution of each edge to the absorption coefficient has been isolated by extrapolating the preedge region to higher energies by a Victoreen-like fit and by substracting the fitted curves from the remaining experimental spectrum [9]. EXAFS data analysis for $M n$ and $S e K$ edges was done applying the well known data reduction procedure described elsewhere [10]. In this work we are mainly interested in neighbor distribution in the first shell. The first shell data was filtered from fine structure using Fourier and back Fourier transform. The one shell parameters $(N, R$ and $\sigma)$ was obtained by non-linear least-squares fitting. Figure 1 shows the $M n-S e$ coordination as a function of $M n$ composition

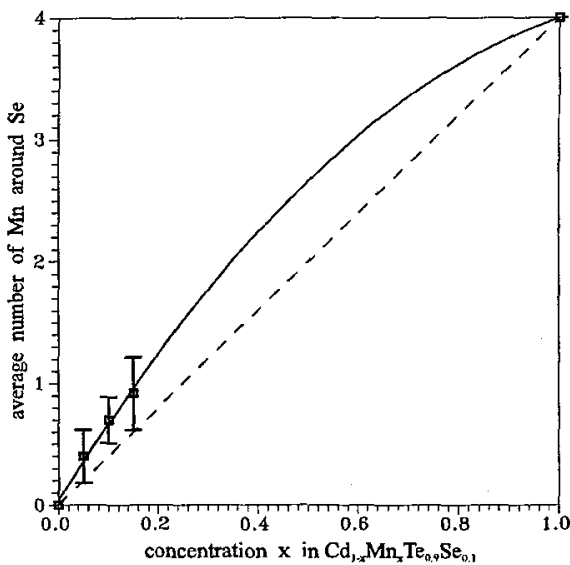

Figure 1: Se neighborhood vs. Mn concentration 
taken from $S e K$-edge. The solid line (square function) was fitted to experimental points for $5 \%, 10 \%, 15 \%$ of $M n$ with $10 \%$ of $S e$ as well as to two border points for ternaries $C d T e_{0.9} S e_{0.1}$ and hypothetical zinc-blende $M n T e_{0.9} S e_{0.1}$ compounds. The consistent deviation from random distribution represented by dashed line is visible. This results are confirmed also by data obtained from $M n K$-edge. This nonlinear dependence is analytically described by quadratic function. Taking into consideration the random distribution background expressed by $4 x$ (dashed line on fig.1) the preference of the site occupation could be approximate as proportional to $x(1-x)$. This result is in very good agreement with Perkowitz et al. [11] expectations for the analytical dependence of the clustering parameter versus composition. For both $S e$ and $M n K$-edges the preference of $M n$-Se pairing over $C d-S e$ and $M n$-Te pairs was observed, respectively. The preference of the distribution of the $\mathrm{Mn}$ ion around Se is in agreement with tendencies anticipated from the lattice constants of the zinc blende binary compounds which fulfill unequality $C d T e>M n T e>C d S e>M n S e$. Also a local ordering in this alloy would be the strain energy which depends on the size of constituent atoms.

In a previous paper [12] we have generalized the model of Czyzyk et al. [2] to the case of quaternary alloys. The model is based entirely on the elastic bond-stretching and bond-bending forces and gives a satisfactory account for nearest-neighbor distances in these alloys. On the other hand, this very simple model does not describe properly the possibility of preferences.

Summarizing, we conclude that apart from the evident structural reason of the preferences which should be discussed in more advanced form, in the case of $C d M n T e S e$ the magnetic superexchange interaction which is larger in CdMnSe than in CdMnTe should be also considered [13].

\section{Acknowledgments}

We wish to thank the technical staff of the PWA group of the Laboratori Nazionali di Frascati for their help and hospitality. This paper has been partially supported by the Polish Committee for Scientific Research (KBN), grant number PB-1147/P3/94/06. We gratefully thank Dr. J. Konior for very stimulating discussions.

\section{References}

[1] A. Balzarotti, N. Motta, A. Kisiel, M. Zimnal-Starnawska, M. T. Czyżyk and M. Podgórny, Phys. Rev. B 31 (1985) 7526.

[2] M. T. Czyżyk, M. Podgórny, A. Balzarotti, P. Letardi, N. Motta, A. Kisiel and M. Zimnal-Starnawska, Z. Phys. B Condensed Matter 62 (1986) 153.

[3] A. Balzarotti, M. T. Czyżyk, A. Kisiel, P. Letardi, N. Motta, M. Podgórny and M. Zimnal-Starnawska, Festkörperprobleme XXV (1985) 689.

[4] M. R. Wiedrnann, J. R. Gregg and K. E. Newman, J. Phys. Condensed Matter 4 (1992) 1895.

[5] W.-F. Pong, R. A. Mayanovic, B. A. Bunker, J. K. Furdyna and U. Debska, Phys. Rev. B 41 (1990) 8440.

[6] S. M. Islam and B. A. Bunker, Physics Letters A 156 (1991) 247.

[7] E. Burattini, E. Bernieri, A. Balerna, C. Mencuccini, R. Rinzivillo, G. Dalba and P. Fornasíni, Nucl. Instrum. Methods A 246 (1986) 125.

[8] S. Chehab, G. Lamarche, A. Manoogian and J. C. Woolley, Journal of Magnetism and Magnetic Materials 59 (1986) 105.

[9] B. K. Teo, EXAFS: Basic Principles and Data Analysis (Springer Verlag, Berlin 1986) pp.

[10] F. W. Lytle, D. E. Sayers and E. A. Stern, Physica B 158 (1989) 701.

[11] S. Perkowitz, L. S. Kim and P. Becla, Phys. Rev. B 43 (1991) 6598.

[12] J. Konior, J. Łażewski, and A. Kisiel, "Microscopic Model of Non-Random Quaternary Alloys", $3^{\text {rd }}$ International School and Symposium on Synchrotron Radiation in Natural Science, Jaszowiec 31 May - 8 June 1996, accepted to publication in Acta Physica Polonica.

[13] A. Lewicki, J. Spałek, J. K. Furdyna and R. R. Gałazka, Journal of Magnetism and Magnetic Materials 54-57 (1986) 1221. 